\title{
Crescimento Pós-Traumático no Câncer de Mama: Centralidade de Evento e Coping
}

\author{
João Oliveira Cavalcante Campos ${ }^{1}$ \\ Clara Cela de Arruda Coelho ${ }^{1}$ \\ Clarissa Marceli Trentini ${ }^{1}$ \\ ${ }^{1}$ Universidade Federal do Rio Grande do Sul, Porto Alegre, Brasil
}

\begin{abstract}
Resumo
Crescimento pós-traumático (CPT) refere-se à mudança positiva em algum aspecto da experiência humana como resultado do enfrentamento de adversidades. Investigou-se a relação entre CPT, estilos de enfrentamento e centralidade de evento. Participaram do estudo 65 mulheres que concluíram os tratamentos recomendados para o câncer de mama. Tratou-se de uma pesquisa quantitativa cujos instrumentos foram respondidos on-line. Identificou-se correlações altas entre CPT e centralidade de evento e moderadas entre CPT e os estilos de enfrentamento: estratégia focada no problema, busca de suporte social e práticas religiosas. A centralidade de evento e as estratégias focadas no problema mostraram-se melhores preditoras de CPT. Os resultados sugerem que quanto maior a adversidade percebida, maior a possibilidade de crescimento, sendo as estratégias de enfrentamento focadas no problema um componente importante para a sua ocorrência. Este estudo apontou a possibilidade de crescimento pessoal relacionado ao enfrentamento do CA de mama e indicou estratégias relevantes para desenvolvê-lo.

Palavras-chave: autorrealização, neoplasias mamárias, memória autobiográfica, estilos de enfrentamento.
\end{abstract}

Post-Traumatic Growth in Breast Cancer: Event Centrality and Coping

\begin{abstract}
Posttraumatic growth (PTG) refers to a positive change in some aspect of the human experience as a result of coping with adversity. This study investigated the relationship between PTG, coping styles, and event centrality, using a cross-sectional research design. The sample consisted of 65 women who had completed the recommended treatments for breast cancer (BC) and answered an online survey. High correlations were identified between PTG and event centrality, and moderate correlations between PTG and the coping styles 'problem-focused strategy', 'social support seeking', and 'religious practices'. Event centrality and problem-focused coping strategies were the best predictors of PTG. The results suggest that the greater the perceived adversity, the greater the possibility of growth, and that problem-focused coping strategies are important for the occurrence of growth in the context of BC. This study pointed to the possibility of personal growth from coping with BC and indicated effective strategies to develop it.

Keywords: self-actualization; breast neoplasms; autobiographical memory; coping styles.
\end{abstract}

Crecimiento Post-Traumático em Cáncer de Mama: Centralidad de Evento y Coping

\section{Resumen}

El crecimiento postraumático (CPT) se refiere al cambio positivo en algún aspecto de la experiencia humana como resultado del enfrentamiento de adversidades. Se investigó la relación entre CPT, estilos de enfrentamiento y centralidad de eventos. Participaron del estudio 65 mujeres que completaron los tratamientos recomendados para el cáncer de mama. Se trató de una investigación cuantitativa respondida on-line. Se identificaron altas correlaciones entre CPT y centralidad de eventos; y moderadas entre CPT y los estilos de enfrentamiento: estrategia centrada en el problema, búsqueda de apoyo social y prácticas religiosas. La centralidad de eventos y las estrategias centradas en el problema demostraron mejores predictores del CPT. Los resultados sugieren que cuanto mayor es la adversidad percibida, mayor es la posibilidad de crecimiento, siendo las estrategias de afrontamientos centradas en el problema un componente importante para su ocurrencia. Este estudio señaló la posibilidad de crecimiento personal frente a la lucha contra el cáncer de mama.

Palabras clave: autorrealización; neoplasias mamárias; memória autobiográfica; estilos de enfrentamiento.

\section{Introdução}

O câncer (CA) de mama é o quarto mais comum no Brasil, representando 15\% do total dos casos de câncer no país estimados para o biênio 2018/2019 (INCA, 2018). Esse tipo de CA acomete majoritariamente mulheres, sendo o tipo mais comum nessa população, excetuando-se os tumores de pele não melanoma. Ainda, é o principal causador de morte por CA na população feminina (INCA, 2018). No Brasil, em 2019, estima-se que houve cerca de 59.700 mil novos casos de câncer de mama, com aproximadamente 51 casos para cada 100 mil mulheres (INCA, 2018).

\section{Aspectos Psicológicos do Enfrentamento do CA de Mama}

Associados ao desconforto físico decorrente do tratamento invasivo - como perda de cabelo, náuseas, vômito e retirada da mama - aspectos emocionais 
estão fortemente envolvidos no enfrentamento da doença. A literatura aponta a ocorrência de emoções negativas, como estresse, medo, raiva, culpa e angústia durante o enfrentamento do CA de mama, sendo recorrente também a presença de transtornos psicológicos, como depressão e ansiedade generalizada (Maluf, Mori, \& Barros, 2005; Vieira, Lopes, \& Shimo, 2007). Provavelmente, parte desse sofrimento deve-se ao fato de o CA de mama alojar-se em um órgão repleto de significados para as mulheres, sendo símbolo de feminilidade, maternidade e sensualidade, por exemplo (INCA, 2015). Nesse contexto, a presença do psicólogo pode ser muito importante, visto que é marcado pelo desconforto do tratamento, sofrimento psicológico e confronto com a possibilidade da morte.

Atualmente, a partir dos esforços para a realização dos diagnósticos precoces e do aumento da eficiência dos tratamentos, o número de sobreviventes ao CA de mama tem crescido. Diversos estudos utilizando amostras com essa população têm demonstrado resultados aparentemente intrigantes: além do sofrimento enfrentado, identifica-se também a existência do relato de crescimento pessoal obtido a partir do enfrentamento da doença (Brunet, McDonough, Hadd, Crocker, \& Sabiston, 2010; Kolokotroni, Anagnostopoulos, \& Tsikkinis, 2014; Parikh et al., 2015; Prati \& Pietrantoni, 2009). Nesse sentido, verifica-se que emoções negativas e transtornos psicológicos não representam a totalidade da experiência de pacientes que enfrentaram o câncer de mama, que também envolve, muitas vezes, um processo de crescimento pessoal.

No Brasil, no entanto, ainda são escassos os estudos que investiguem essa perspectiva. Diante disso, sublinha-se a importância de se estudar o conceito de crescimento pós-traumático (CPT) para entender a experiência de sobreviventes ao $\mathrm{CA}$ de mama que lograram uma readaptação bem-sucedida (Araújo, 2013). Apesar do relato de crescimento pessoal não ser comum a todas as sobreviventes, investigá-lo pode contribuir na elaboração de intervenções que se proponham a promovê-lo.

\section{Crescimento Pós-Traumático, Centralidade de Evento e Coping}

O conceito de CPT foi desenvolvido por Tedeschi \& Calhoun (1996) e refere-se à mudança positiva em algum aspecto da experiência humana como resultado do enfrentamento de situações estressoras ou traumáticas. Os pesquisadores estão alinhados à proposta da Psicologia Positiva (Seligman \& Csikszentmihalyi, 2014) e fundamentam-se no argumento de que, até então, se havia investigado extensivamente os efeitos negativos de eventos traumáticos e, por outro lado, pouca atenção havia sido dada aos impactos psicológicos positivos decorrentes do enfrentamento de tais eventos.

O crescimento advindo de situações traumáticas tem sido investigado em vítimas de ataque cardíaco (Karagiorgou \& Cullen, 2016), sobreviventes de abuso sexual na infância (Hartley, Johnco, Hofmeyr, \& Berry, 2016), veteranos de guerra (Pietrzak \& Tsai, 2017), pessoas que vivenciaram luto (Zhou, Yu, Tang, Wang, \& Killikelly, 2018), pacientes com HIV (Rzeszutek \& Gruszczyńska, 2018), pais de crianças com doenças de alto risco (Nakayama et al., 2016), vítimas de queimaduras graves (Martin, Byrnes, McGarry, Rea, \& Wood, 2017), sobreviventes de desastres naturais (Taku et al., 2015), sobreviventes do holocausto (Lev-Wiesel \& Amir, 2003), pacientes com câncer (Cormio, Muzzatti, Romito, Mattioli, \& Annunziata, 2017) e também em sobreviventes ao CA de mama (Ramos et al., 2018).

Segundo Tedeschi \& Calhoun (2004), o CPT teria características específicas: (a) acontece no contexto de enfrentamento de uma situação caracterizada por um alto grau de estresse; (b) trata-se de um crescimento real e verídico, em oposição a uma ilusão perceptiva; (c) não se refere a uma forma específica de encarar uma situação (coping), aproximando-se mais de um resultado contínuo desse processo e, (d) para que aconteça esse tipo de crescimento, é necessário que crenças fundamentais dos indivíduos sejam ameaçadas ou abaladas, de modo que, muitas vezes, ele ocorre concomitantemente com a presença de estresse psicológico.

Segundo a visão de Janoff-Bulman (2004), uma situação traumática seria aquela que abalaria as regras e crenças centrais do indivíduo em relação a sua visão de si e/ou do mundo (e.g., a vida como sendo boa e justa), o que envolveria bastante estresse psicológico. Dispondo de recursos adaptativos momentaneamente limitados para lidar com a situação, ocorreria inicialmente no indivíduo um processo de ruminação intrusiva (Janoff-Bulman, 2004), que seria caracterizado por envolver pensamentos repetitivos, involuntários e focados no sofrimento e em suas possíveis causas e consequências (Nolen-Hoeksema, Wisco, \& Lyubomirsky, 2008). Gradativamente, o processo de ruminação intrusiva poderia dar lugar a um tipo de ruminação deliberada, caracterizada por envolver pensamentos intencionais, que podem facilitar a construção de sentido, assimilação do evento e CPT (Jim \& Jacobsen, 2008; Tedeschi \& Calhoun, 2004). Nessa perspectiva, a ruminação de informações 
traumáticas, inicialmente de modo automático e gradualmente de modo mais deliberado, refletiria a ativação de processamento cognitivo, direcionado ao objetivo de reconstruir as crenças básicas dos indivíduos, de forma a assimilar a situação experienciada (Janoff-Bulman, 2004; Tedeschi \& Calhoun, 2004).

Trata-se, portanto, de uma perspectiva cognitivista, na qual não é o evento em si que determina se uma situação é traumática ou não para o indivíduo, mas sim como ele a vivencia subjetivamente, ou seja, qual o seu impacto sobre as crenças centrais dos indivíduos. Assim, alguns indivíduos, após terem vivenciado uma situação traumática, gradativamente (ao longo do tempo) conseguiriam processar cognitivamente a situação e integrar a experiência traumática a sua história de vida, podendo, então, relatar mudanças positivas (Tedeschi \& Calhoun, 1996; Tedeschi \& Calhoun, 2004).

Tem-se obtido evidências de que, quanto maior o impacto psicológico do evento experienciado, maior a possibilidade de o indivíduo relatar a ocorrência de CPT (Boals \& Schuettler, 2011; Johnson \& Boals, 2015). Nesse sentido, estudos recentes têm mostrado a importância de se considerar a centralidade do evento adverso como forma de avaliar o seu impacto psicológico no indivíduo (Groleau, Calhoun, Cann, \& Tedeschi, 2013; Johnson \& Boals, 2015). O construto centralidade de evento refere-se ao quanto a memória de um evento estressor vivenciado é um marco na vida do indivíduo, o quanto contribuiu na formação da sua identidade e para a interpretação de outros eventos na sua vida (Berntsen \& Rubin, 2006; Gauer, de Souza, da Silveira, \& Sediyama, 2013).

Parikh et al. (2015) realizaram uma revisão sistemática de 47 artigos publicados entre 1985 e 2014, envolvendo estresse pós-traumático e CPT no CA de mama. Os resultados indicaram que apenas um percentual pequeno das sobreviventes apresentou estresse pós-traumático, enquanto a maioria apresentou CPT, o que indica a importância de se estudar a experiência dessas mulheres. Além disso, nesse contexto, tem-se identificado a relevância das estratégias de enfrentamento ou coping para o desenvolvimento de CPT nessa população (Danhauer et al., 2013; Hamama-Raz, Pat-Horenczyk, Roziner, Perry, \& Stemmer, 2019; Kolokotroni et al., 2014; Pat-Horenczyk et al., 2015).

Coping refere-se ao conjunto de estratégias que as pessoas utilizam para o enfrentamento de situações difíceis (Antoniazzi, Dell'Aglio, \& Bandeira, 1998; Folkman, Lazarus, Dunkel-Schetter, DeLongis, \& Gruen,
1986). Apesar de haver muitas variações de nomenclaturas a respeito das diferentes estratégias de coping, elas podem ser classificadas em categorias. Em geral, pode-se falar em estratégias de coping ativas/engajadas e estratégias de coping evitativas (Danhauer et al., 2013; Stanton, Bower, \& Low, 2006).

Em estudo longitudinal, realizado por Danhauer et al. (2013), 653 mulheres diagnosticadas com CA de mama foram acompanhadas por mais de dois anos. Os resultados apontaram para a relevância das estratégias ativas/engajadas de coping para o desfecho de CPT. Além disso, uma meta-análise analisou 103 estudos envolvendo a investigação de variáveis associadas ao CPT (Prati \& Pietrantoni, 2009). Nesse trabalho, identificou-se a relevância dos seguintes estilos de coping: estratégias religiosas, reinterpretação positiva e busca por suporte social. No Brasil, no entanto, estudos envolvendo CPT, estratégias de coping e CA de mama ainda são escassos.

No presente estudo, considerou-se os seguintes estilos de coping (Seidl, Tróccoli, \& Zannon, 2001): (a) estratégias focalizadas no problema (adaptativas), que se referem a formas de engajamento na modificação da situação causadora de estresse, visando à solução de problemas; (b) estratégias focalizadas na emoção (desadaptativas), que buscam a regulação da resposta emocional, causada pelo enfrentamento do problema, podendo representar atitudes de afastamento, como negação ou esquiva; (c) busca por suporte social, que envolve a busca por apoio emocional e instrumental no enfrentamento da doença; e (d) práticas ou crenças religiosas, que envolvem uma forma espiritualizada de dar sentido e lidar com o problema.

De acordo com o exposto, evidencia-se a necessidade de se estudar CPT, particularmente, na população de mulheres sobreviventes ao CA de mama, e de se identificar fatores possivelmente associados a ele. $\mathrm{O}$ presente trabalho tem o objetivo principal investigar o CPT em mulheres que concluíram os tratamentos indicados para CA de mama e que, atualmente, não apresentam mais sinais da doença. Os objetivos específicos são (a) investigar a relação entre CPT e a centralidade de evento (adoecimento e tratamento); (b) investigar a relação entre CPT e estilos de coping; (c) investigar a relação entre CPT e variáveis sociodemográficas e clínicas (idade, tempo decorrido desde o diagnóstico, prática de atividade física, estadiamento da doença, procedimentos realizados) e (d) identificar possíveis preditoras de CPT dentre as variáveis investigadas. 


\section{Método}

\section{Participantes}

Participaram do estudo 65 mulheres com idade média de 47,86 anos $(D P=8,43)$, variando de 28 a 64 anos, que concluíram os tratamentos indicados para CA de mama (mastectomia, quimioterapia e/ou radioterapia) e não apresentavam mais sinais da doença. Desse grupo, $67 \%$ residem na região Sudeste, 50,80\% possuem ensino superior completo, $43 \%$ trabalham e 65,6\% são casadas ou possuem outra forma de união. O tempo médio relatado desde o diagnóstico foi de 3,68 $\operatorname{anos}(D P=2,96)$.

\section{Instrumentos}

Foram aplicados os seguintes instrumentos:

Questionário Sociodemográfico e Clínico. Contém perguntas referentes à caracterização sociodemográfica da amostra (como idade, escolaridade, renda e estado civil) e aos aspectos clínicos durante o curso da doença (como idade no período do diagnóstico, estágio da doença e tratamentos realizados).

Escala de Centralidade de Eventos (Berntsen \& Rubin, 2006; Gauer et al., 2013). O instrumento mede o quanto a memória de um evento estressor vivenciado é um marco na vida do indivíduo, o quanto contribuiu na formação da sua identidade e para a interpretação de outros eventos na sua vida. É composto por 20 itens, respondidos por meio de uma escala Likert de cinco pontos. A versão brasileira do instrumento apresentou adequado índice de fidedignidade $\alpha=0,95$. No presente estudo, o instrumento foi utilizado como uma medida do impacto psicológico do CA de mama (diagnóstico e tratamento).

Inventário de Crescimento Pós-Traumático (Tedeschi \& Calhoun, 1996). A versão brasileira do instrumento conta com 18 itens e é respondida por meio de uma escala Likert de seis pontos para o grau de mudanças apresentadas após uma crise específica. Foi adaptado e validado para o uso no Brasil (Silva, Donat, Gauer, \& Kristensen, 2016; Silva et al., 2018). Obteve-se uma estrutura fatorial semelhante à original, composta pelas dimensões: relacionamento com os outros ( 6 itens; $\alpha=0,84$ ), novas possibilidades ( 5 itens; $\alpha=0,83$ ), força pessoal ( 3 itens; $\alpha=0,70$ ), apreciação da vida ( 2 itens; $\alpha=0,81$ ) e mudança espiritual ( 2 itens; $\alpha=0,85)$. A escala geral também apresentou índice de fidedignidade adequado $(\alpha=0,91)$, assim com as subescalas. Além disso, um estudo recentemente realizado no contexto brasileiro indicou ser adequada a utilização de uma dimensão geral de CPT (Campos \& Trentini, 2019).

Escala Modos de Enfrentamento de Problemas (Seidl et al., 2001; Vitalino, Russo, Carr, Maiuro, \& Becker, 1985). A escala foi adaptada para o Brasil por Gimenes \& Queiroz (1997) e validada por Seidl, Tróccoli e Zannon (2001). É composta por 45 itens divididos em quatro subescalas: estratégias focalizadas no problema (adaptativas) (18 itens; $\alpha=0,84)$, estratégias focalizadas na emoção (desadaptativas) (15 itens; $\alpha$ $=0,81$ ), práticas religiosas ( 7 itens; $\alpha=0,74$ ) e busca de suporte social ( 5 itens; $\alpha=0,70)$.

\section{Delineamento e Procedimento de Coleta}

Tratou-se de um estudo com delineamento transversal. A coleta de dados foi realizada on-line, por meio da plataforma SurveyMonkey. O convite para a participação foi divulgado por meio de uma rede social, dentro de grupos fechados de apoio a pacientes com CA de mama. Primeiramente, foi feito um contato individual com a responsável por cada grupo de apoio às pacientes, esclarecendo a respeito da pesquisa e solicitando a permissão para divulgá-la. O convite a participação foi direcionado às pacientes que concluíram os tratamentos para o CA de mama e não apresentavam mais sinais da doença. A resposta aos questionários levou em média 30 minutos.

O presente estudo foi submetido ao Comitê de Ética em Pesquisa e foi aprovado, sob o protocolo número 1.676 .037 , de acordo com a resolução 510/2016 do Conselho Nacional de Saúde. Somente as participantes que concordaram com o Termo de Consentimento Livre e Esclarecido foram apresentadas aos questionários referentes à pesquisa.

\section{Procedimento de Análise de Dados}

Por meio do software IBM SPSS Statistics (versão 18), foram realizadas as estatísticas descritivas para as variáveis de interesse. Além disso, foram realizados testes $t$ e correlações de Pearson para investigar a relação entre CPT e variáveis sociodemográficas e clínicas, centralidade de evento e estilos de coping.

Posteriormente, testou-se modelos de regressão linear (método forward), tendo como possíveis variáveis explicativas a centralidade de evento e os estilos de coping, e como variável de desfecho, o CPT. Esse método de análise de variáveis é utilizado para situações em que se busca investigar os dados de forma exploratória (Field, 2009). De acordo com esse método, a primeira variável preditora que entra no modelo de regressão é 
aquela que melhor prevê a variável de desfecho. Nas etapas seguintes, os demais preditores são incorporados sucessivamente, de acordo com dois critérios: 1) possuir maior coeficiente de correlação semiparcial com a variável de desfecho e 2) aumentar de forma estatisticamente significativa a capacidade preditiva do modelo. As variáveis que não atendem a esses critérios não são incorporadas ao modelo.

\section{Resultados}

Os dados sociodemográficos e as características clínicas da amostra foram descritos na Tabela 1 e na Tabela 2, respectivamente. Os resultados de correlações e dos testes utilizados para comparar os níveis de CPT em subgrupos da amostra foram descritos a seguir. Não foram encontradas correlações estatisticamente significativas entre idade durante o diagnóstico $(r=-0,2, n=$ $60, p=0,85)$ e tempo decorrido desde o diagnóstico $(r=$ $0,09, n=58, p=0,50)$ frente à variável CPT. Para investigar o impacto do estágio da doença, ao momento do diagnóstico, nos níveis de CPT, subdividiu-se a amostra

Tabela 1.

Dados Sociodemográficos

\begin{tabular}{lc}
\hline Variáveis & $\%$ \\
\hline Escolaridade & 9 \\
\hline Ensino Fundamental & 28,4 \\
Ensino Médio & 37,3 \\
Ensino Superior & 25,4 \\
Pós-Graduação & \\
\hline Estado Civil & 65,6 \\
\hline Casada/união estável & 14,9 \\
Divorciada/separada & 4,5 \\
Viúva & 11,9 \\
Solteira & \\
\hline Renda familiar & 13,8 \\
\hline Até dois salários mínimos & 33,8 \\
2 a 4 salários mínimos & 24,6 \\
4 a 10 salários mínimos & 20 \\
10 a 20 salários mínimos & 7,7 \\
Acima de 20 salários mínimos & \\
Atividade física regular & 53 \\
Sim & 47 \\
Não
\end{tabular}

em dois grupos: baixa gravidade (estágios I e II) e alta gravidade (estágios III, IV). No entanto, não foi encontrada diferença estatisticamente significativa nos níveis de CPT entre o grupo baixa gravidade $(M=4,65 ; D P=$ $1,57)$ e o grupo alta gravidade $(M=5,02 ; D P=0,58)$, $\mathrm{t}(25,23)=-1,059, p=0,30$.

Não foi encontrada diferença estatisticamente significativa entre o grupo de grupo de mulheres que realizaram os procedimentos de mastectomia (uma ou duas mamas) ( $M=4,95 ; D P=1,02)$ e o grupo de mulheres que não realizaram $(M=4,83 ; D P=1,10)$, $\mathrm{t}(59)=-0,420, p=0,68$. Também não foi encontrada diferença estatisticamente significativa nos níveis de CPT entre quem pratica $(M=4,95 ; D P=1,05)$ e quem não pratica atividade física regularmente $(M=4,85 ; D P$ $=1,05), \mathrm{t}(59)=0,345, p=0,73$.

As participantes indicaram, numa escala de 1 a 10, a gravidade percebida, de "nada grave" à "extremamente grave", uma média de 7,5(DP $=2,4)$ para a situação vivenciada. Ainda, 86,5\% das participantes relataram gravidade percebida entre 5 e 10 . Não foram observadas diferenças estatisticamente significativas nos níveis de CPT entre os grupos que tiveram e os que não tiveram atendimento psicológico/psiquiátrico. No entanto, todas as participantes que contaram com esse tipo de acompanhamento $(N=20)$, relataram que este contribuiu positivamente para o enfrentamento da doença.

$\mathrm{Na}$ Tabela 3, foram descritas médias, desvios padrões e correlações de Pearson entre as variáveis de

Tabela 2.

Variáveis Clínicas

\begin{tabular}{lc}
\hline Estágio do câncer & $\%$ \\
\hline I & 4,8 \\
II & 32,3 \\
III & 29 \\
IV & 21 \\
Procedimentos realizados & $\%$ \\
Mastectomia (1 mama) & $49,3 \%$ \\
Mastectomia (2 mamas) & $14,9 \%$ \\
Tumorectomia & $1,5 \%$ \\
Quadrantectomia & $32,8 \%$ \\
Quimioterapia & $86,6 \%$ \\
Radioterapia & $76,1 \%$ \\
Terapêutica hormonal & $41,8 \%$ \\
\hline
\end{tabular}


interesse. Foram observadas correlações altas entre CPT e centralidade de evento. Além disso, foram identificadas correlações estatisticamente significativas moderadas entre estratégias de coping focadas no problema, busca de suporte social, práticas religiosas e CPT. Não foram obtidas correlações estatisticamente significativas entre estratégias de coping focadas em emoções (desadaptativas) e CPT.

$\mathrm{Na}$ Tabela 4, foram descritos indicadores referentes à regressão linear múltipla, utilizando-se o método forward para a entrada das variáveis no modelo. As variáveis que se correlacionaram positivamente com CPT (centralidade de evento, estratégias focadas no problema, busca por suporte social e práticas religiosas) foram incluídas como possíveis preditoras de CPT.

$\mathrm{Na}$ Etapa 1, a variável centralidade de evento manteve-se no modelo, $\operatorname{com} \mathrm{F}(1,47)=27,044, p<0,01 . \mathrm{Na}$ Etapa 2, a variável estratégias focadas no problema foi incorporada ao modelo acrescentando poder explicativo, $\operatorname{com} \mathrm{F}(2,47)=26,997, p<0,01$. Apesar de ambos os modelos gerados (na Etapa 1 e na Etapa 2) terem se mostrado estatisticamente significativos, o modelo disposto na Etapa 2 foi o que explicou um maior percentual da variância em CPT (52\%). Nele, as variáveis centralidade de evento e estratégias de coping focadas no problema foram incorporadas como melhores preditoras de CPT. Após a inclusão de ambas, as variáveis busca por suporte social e práticas religiosas não contribuíram de forma estatisticamente significativa com a capacidade do modelo de prever o CPT, sendo, portanto, descartadas.

\section{Discussão}

$\mathrm{Na}$ amostra investigada, as variáveis sociodemográficas e clínicas (prática de atividade física, idade durante o diagnóstico, tempo decorrido desde o diagnóstico, estadiamento da doença e tipo de tratamento realizado) não mostraram relevância estatística quando se investigou sua influência nos níveis de CPT. Tais resultados devem ser tomados com cautela, uma vez que podem refletir a influência de variáveis intervenientes,

Tabela 3.

Correlacões entre CPT e Variáveis do Estudo

\begin{tabular}{lccccccc}
\hline Variáveis & $\mathbf{1}$ & $\mathbf{2}$ & $\mathbf{3}$ & $\mathbf{4}$ & $\mathbf{5}$ & $\mathbf{M}(\boldsymbol{D P})$ & Intervalo \\
\hline CPT & & & & & $4,90(1,04)$ & $1-6$ \\
ECE & $0,60^{* *}$ & & & & $3,82(0,92)$ & $1-5$ \\
EF_P & $0,47^{* *}$ & 0,20 & & & & $3,94(0,49)$ & $1-5$ \\
EF_E & 0,30 & $0,27^{*}$ & $-0,25$ & & & $1,99(0,65)$ & $1-5$ \\
Busca_SS & $0,40^{* *}$ & $0,34^{*}$ & $0,55^{* *}$ & 0,20 & & $3,26(0,62)$ & $1-5$ \\
P_Rel & $0,32^{*}$ & 0,015 & $0,34^{* *}$ & 0,21 & $0,34 * *$ & $3,69(0,78)$ & $1-5$ \\
\hline
\end{tabular}

Nota. CPT $=$ Crescimento Pós-Traumático; ECE = Escala de Centralidade de Evento; EF_P = Estratégias Focadas no Problema; EF_E = Estratégias Focadas na Emoção (desadaptativas); Busca_SS = Busca por Suporte Social; P_Rel = Práticas Religiosas. ${ }^{*} p \leq 0,05 ; * * p \leq 0,001$

Tabela 4.

Modelos de Regressão Linear

\begin{tabular}{lccccc}
\hline CPT & B & SE B & B Padronizado & $\mathrm{R}^{2}$ Ajustado & $\Delta \mathrm{R}^{2}$ \\
\hline Etapa 1 & & & & $0,36^{*}$ & \\
ECE & 0,71 & 0,14 & $0,61^{*}$ & & $0,52^{*}$ \\
Etapa 2 & & & & & \\
ECE & 0,62 & 0,12 & $0,54^{*}$ & \\
EF_P & 0,93 & 0,22 & $0,42^{*}$ & & \\
\hline
\end{tabular}

Nota. CPT = Crescimento Pós-Traumático; ECE = Escala de Centralidade de Evento; EF_P = Estratégias Focadas no Problema; $* p \leq 0,01$. 
decorrentes da alta heterogeneidade da amostra (em aspectos clínicos e socioeconômicos). Além disso, a influência de variáveis sociodemográficas e clínicas para o CPT no contexto do CA de mama ainda não está bem estabelecida na literatura, uma vez que existem ainda poucas evidências nesse sentido (Kolokotroni et al., 2014).

De todo modo, os resultados do presente estudo, em síntese, corroboram a perspectiva de que, no que se refere à possibilidade de CPT, aspectos subjetivos relacionados ao enfrentamento da doença têm uma maior influência do que questões objetivas. Em direção semelhante, apontam os achados de Cordova, Cunningham, Carlson e Andrykowski (2001), que não observaram relação estatisticamente significativa entre CPT e variáveis clínicas (estágio da doença durante o diagnóstico e tipo de tratamento) e de Cordova et al. (2007), que não encontraram relação significativa entre tempo desde o diagnóstico e CPT. Do ponto de vista psicológico, a relevância dos aspectos subjetivos para a ocorrência de CPT no contexto do CA de mama é notável, uma vez que são justamente esses que podem ser mais facilmente modificados ou ressignificados, diferentemente de condições socioeconômicas e/ou clínicas.

No presente estudo, ao considerar a gravidade da situação vivenciada, a maioria $(86,5 \%)$ das participantes percebeu a situação enfrentada de moderada à extremamente grave. O impacto psicológico da doença também foi identificado por meio da Escala de Centralidade de Eventos (ECE), na qual 64,2\% da amostra percebeu o impacto psicológico de moderado a alto. Esses dados corroboram a hipótese de que o CA de mama é uma doença que envolve significativo grau de impacto psicológico.

Ainda, obteve-se uma alta correlação entre CPT e a centralidade do evento, que também se mostrou o melhor preditor de CPT no modelo de regressão elaborado. O impacto do diagnóstico e tratamento do CA de mama pode estar relacionado com a confrontação da própria mortalidade, podendo resultar em mudança nas prioridades, na apreciação pela vida, nas relações interpessoais, na relação com a espiritualidade e contribuir para a descoberta de novas possibilidades na vida (Brunet et al., 2010). Desse modo, quanto maior o impacto psicológico do evento, maior seria a possibilidade de ocorrer CPT (Boals \& Schuettler, 2011).

Neste estudo, $88,5 \%$ da amostra relatou que experienciou níveis de CPT de grau moderado a muito intenso. Logo, esse resultado apoia a hipótese mencionada de que é possível crescer a partir do enfrentamento de adversidades, como o CA de mama (Parikh et al., 2015). No entanto, no contexto do enfrentamento da doença, conforme sugerem os resultados obtidos por Cordova et al. (2007), é possível a ocorrência tanto de desfechos psicológicos negativos (sintomas de estresse pós-traumático), como de desfechos positivos (CPT) de forma aparentemente independente. Os estilos de coping parecem desempenhar um papel importante para o desenvolvimento do CPT (Schuettler \& Boals, 2011).

No presente trabalho, as estratégias de coping focadas no problema, a busca por suporte social e as práticas religiosas correlacionaram-se positivamente com CPT (correlações moderadas). No que se refere à busca por suporte social e as práticas religiosas, a literatura aponta que se tratam de estratégias de coping relevantes no contexto do CA de mama e que contribuem para o desenvolvimento do CPT (Prati \& Pietrantoni, 2009). Prati e Pietrantoni (2009) sugerem que a busca por suporte social poderia contribuir para aumentar a qualidade e a quantidade do suporte social recebido. Para Tedeschi e Calhoun (2004), o suporte social, além de possibilitar um melhor gerenciamento do estresse por parte do indivíduo, por meio de suporte emocional e instrumental, poderia favorecer à elaboração de narrativas a respeito da situação enfrentada, contribuindo para o processamento cognitivo do evento. Já no que se refere à importância de estratégias de coping religioso, de fato, há evidências de que podem favorecer que o indivíduo, diante do sofrimento, encontre sentido proveniente da crença em um ser superior que o apoia e o ama (Park, 2005), contribuindo para a ocorrência de crescimento pessoal (García, Páez, Reyes-Reyes, \& Álvarez, 2017).

Apesar das estratégias buscar por suporte social e práticas religiosas terem se correlacionado positivamente com CPT, após as estratégias de coping focadas no problema terem sido inclusas na regressão, ambas não acrescentaram poder explicativo ao modelo. Dessa forma, as estratégias de coping focadas no problema mostraram-se mais relevantes do que as demais enquanto preditora de CPT.

As estratégias focadas no problema envolvem a atitude do indivíduo de encarar o problema, buscando realizar os passos necessários para resolvê-lo, assim como uma atitude de reestruturação cognitiva no modo de olhar a situação (Seidl et al., 2001). Stanton, Bower e Low (2006), em uma revisão da literatura sobre estudos envolvendo pacientes com CA e CPT, também identificaram a relevância de estratégias de coping focadas 
no problema enquanto preditoras de CPT. Os autores classificam esse tipo de abordagem como engajada diante do enfrentamento da situação estressora, o que, segundo as evidências encontradas, favorece a ocorrência de CPT, em oposição a uma postura evitativa de lidar com as questões envolvidas no processo de adoecimento/tratamento (Stanton et al., 2006).

No contexto do enfrentamento do CA de mama, ressalta-se a importância de a paciente ter acesso ao conhecimento dos passos que deve seguir ao longo do processo, esclarecendo-se mitos e tabus envolvendo o diagnóstico e o tratamento (Maluf et al., 2005). O acesso à informação sobre a sua condição e o percurso que será percorrido, possivelmente pode ajudá-la a adotar uma postura menos evitativa e mais engajada no tratamento. Assim, entende-se que o profissional de saúde tem um papel relevante nesse processo, especialmente, o psicólogo, que poderá contribuir ao favorecer a elaboração e ressignificação da situação de adoecimento/tratamento (Maluf et al., 2005). De fato, no presente estudo, todas as pacientes que tiveram acompanhamento psicológico relataram a sua contribuição para um melhor enfrentamento da doença.

Ressalta-se que as estratégias baseadas nas emoções desadaptativas não apresentaram correlações estatisticamente significativas com CPT. Esse estilo de coping envolve soluções paliativas no enfrentamento da situação problema (como negação, raiva, culpa e isolamento) (Seidl et al., 2001). Provavelmente, ele está associado a processos ruminativos intrusivos e, portanto, não contribui diretamente para a ocorrência de CPT. As estratégias de enfrentamento baseadas nas emoções desadaptativas, em média, foram as menos utilizadas pelas participantes do presente estudo, no entanto, tratam-se de reações naturais durante o enfrentamento da doença. As diferentes estratégias de coping não são excludentes, muitas vezes elas ocorrem simultaneamente (Folkman et al., 1986). No entanto, seria interessante para a paciente que, aos poucos, as estratégias desadaptativas fossem menos utilizadas e as estratégias mais adaptativas ganhassem espaço.

$\mathrm{O}$ presente estudo contribuiu no sentido de investigar o CPT em uma amostra de mulheres brasileiras que enfrentaram o CA de mama. Nesse contexto, identificou-se como variáveis preditoras do CPT, a centralidade de evento e as estratégias de coping focadas no problema.

No entanto, ressalta-se que a concepção amplamente difundida de que um evento traumático resulta apenas em desordens não deve ser substituída pela noção simplista de que o crescimento é um resultado inevitável, tendo em vista que, muitas vezes, o estresse e o crescimento coexistem e, em alguns casos, não se observa relato de crescimento pessoal após a vivência de uma experiência adversa (Tedeschi \& Calhoun, 2004).

Este estudo transversal utilizou uma amostra relativamente reduzida e com uma heterogeneidade de condições clínicas enfrentadas. Portanto, os achados devem ser interpretados com cautela. Pesquisas futuras podem beneficiar-se do uso de amostras maiores e com condições mais controladas, possibilitando uma maior confiabilidade nos achados. Idealmente, para o desenvolvimento dos conhecimentos na área, é interessante o desenvolvimento de estudos que utilizem um delineamento longitudinal, com o uso de medidas repetidas desde o diagnóstico, possibilitando o acompanhamento mais detalhado da evolução das pacientes. Além disso, pesquisas qualitativas podem auxiliar na melhor compreensão do fenômeno a partir do aprofundamento das experiências individuais das mulheres. Apesar de suas limitações, este estudo traz contribuições para a ampliação do entendimento da vivência do CA de mama entre mulheres, especialmente, ao apontar a possibilidade de crescimento pessoal a partir dessa situação potencialmente traumática e indicar possíveis estratégias que facilitem um desfecho positivo.

\section{Referências}

Antoniazzi, A. S., Dell'Aglio, D. D., \& Bandeira, D. R. (1998). O conceito de coping: Uma revisão teórica. Estudos de Psicologia (Natal), 3(2), 273-294. doi: 10.1590/S1413-294X1998000200006

Araújo, L. T. (2013). Crescimento pós-traumático em mulheres acometidas por câncer de mama: Revisão sistemática da literatura (dissertação de mestrado não publicada). Instituto de Psicologia da Universidade Federal de Uberlândia, Uberlândia, Brasil.

Berntsen, D., \& Rubin, D. C. (2006). The centrality of event scale: A measure of integrating a trauma into one's identity and its relation to posttraumatic stress disorder symptoms. Behaviour Research and Therapy, 44(2), 219-231. doi: 10.1016/j. brat.2005.01.009

Boals, A., \& Schuettler, D. (2011). A double-edged sword: Event centrality, PTSD and posttraumatic growth. Applied Cognitive Psychology, 25(5), 817-822. doi: 10.1002/acp.1753

Psico-USF, Bragança Paulista, v. 26, n. 3, p. 417-428, jul./set. 2021 
Brunet, J., McDonough, M. H., Hadd, V., Crocker, P. R. E., \& Sabiston, C. M. (2010). The posttraumatic growth inventory: An examination of the factor structure and invariance among breast cancer survivors. Psycho-Oncology, 19(8), 830-838. doi: 10.1002/pon. 1640

Campos, J. O. C., \& Trentini, C. M. (2019). Análise fatorial confirmatória da versão brasileira do Inventário de Crescimento Pós-Traumático. Avaliação Psicológica, 18(1), 50-57. doi: 10.15689/ ap.2019.1801.14667.06

Cordova, M. J., Cunningham, L. L., Carlson, C. R., \& Andrykowski, M. a. (2001). Posttraumatic growth following breast cancer: A controlled comparison study. Health Psychology : Official Journal of the Division of Health Psychology, American Psychological Association, 20(3), 176-185. doi: 10.1037/0278-6133.20.3.176

Cordova, M. J., Giese-Davis, J., Golant, M., Kronenwetter, C., Chang, V., \& Spiegel, D. (2007). Breast cancer as trauma: Posttraumatic stress and posttraumatic growth. Journal of Clinical Psychology in Medical Settings, 14, 308-319. doi: 10.1007/ s10880-007-9083-6

Cormio, C., Muzzatti, B., Romito, F., Mattioli, V., \& Annunziata, M. A. (2017). Posttraumatic growth and cancer: A study 5 years after treatment end. Support Care Cancer, 25(4), 1087-1096. doi: 10.1007/ s00520-016-3496-4

Danhauer, S. C., Case, L. D., Tedeschi, R., Russell, G., Vishnevsky, T., Triplett, K., ... Avis, N. E. (2013). Predictors of posttraumatic growth in women with breast cancer. Psycho-Oncology, 22(10), 26762683. doi: $10.1002 /$ pon.3298

Field, A. (2009). Descobrindo a estatística usando o SPSS. Porto Alegre: Artmed.

Folkman, S., Lazarus, R. S., Dunkel-Schetter, C., DeLongis, A., \& Gruen, R. (1986). Dynamics of a stressful encounter: Cognitive appraisal, coping and encounters outcomes. Journal of Personality and Social Psychology, 50(5), 992-1003.

García, F. E., Páez, D., Reyes-Reyes, A., \& Álvarez, R. (2017). Religious coping as moderator of psychological responses to stressful events: A longitudinal study. Religions, 8(62).

Gauer, G., de Souza, J. A., da Silveira, A. M., \& Sediyama, C. Y. N. (2013). Stressful events in autobiographical memory processing: Brazilian version of the Centrality of Event Scale. Psicologia: Reflexao e Critica, 26(1), 98-105. doi: 10.1590/ S0102-79722013000100011

Groleau, J. M., Calhoun, L. G., Cann, A., \& Tedeschi, R. G. (2013). The role of centrality of events in posttraumatic distress and posttraumatic growth. Psychological Trauma: Theory, Research, Practice, and Policy, 5(5). doi: 10.1037/a0028809

Hamama-Raz, Y., Pat-Horenczyk, R., Roziner, I., Perry, S., \& Stemmer, S. M. (2019). Can posttraumatic growth after breast cancer promote positive coping? A cross-lagged study. Psychooncology, 28(4), 767-774. doi: 10.1002/pon.5017

Hartley, S., Johnco, C., Hofmeyr, M., \& Berry, A. (2016). The nature of posttraumatic growth in adult survivors of child sexual abuse. Journal of Child Sexual Abuse, 25(2), 201-220. doi: 10.1080/10538712.2015.1119773

INCA, I. N. de C. J. A. G. da S. (2015). Perfil da assistência oncológica, de 2007 a 2011. Informativo Vigilância do Câncer (Vol. 6). Rio de Janeiro.

INCA, I. N. de C. J. A. G. da S. (2018). Estimativa 2018: Incidência de câncer no brasil. (Coordenação-Geral de Prevenção e Vigilância, Ed.). Rio de Janeiro: Ministério da Saúde e Instituto Nacional de Câncer José Alencar Gomes da Silva (INCA). Recuperado de https://www.inca.gov.br/sites/ufu.sti.inca. local/files// media/document//estimativaincidencia-de-cancer-no-brasil-2018.pdf

Janoff-Bulman, R. (2004). Posttraumatic growth: Three explanatory models. Psychological Inquiry, 15, 30-14.

Jim, H. S. L., \& Jacobsen, P. B. (2008). Posttraumatic stress and posttraumatic growth in cancer survivorship: A review. The Cancer Journal, 14(6), 414-419. doi: 10.1097/PPO.0b013e31818d8963

Johnson, S. F., \& Boals, A. (2015). Refining our ability to measure posttraumatic growth. American Psychological Association, 7(5), 422-429. doi: 10.1037/ tra0000013

Karagiorgou, O., \& Cullen, B. (2016). A Comparison of posttraumatic growth after acquired brain injury or myocardial infarction. Journal of Loss and Trauma, 21(6), 589-600. doi: 10.1080/15325024.2016.1161427 
Kolokotroni, P., Anagnostopoulos, F., \& Tsikkinis, A. (2014). Psychosocial factors related to posttraumatic growth in breast cancer survivors: A review. Women \& Health, 54(6), 569-592. doi: 10.1080/03630242.2014.899543

Lev-Wiesel, R., \& Amir, M. (2003). Posttraumatic growth among holocaust child survivors. Journal of Loss and Trauma, 8(4), 229-237. doi: $10.1080 / 15325020305884$

Maluf, M. F. de M., Mori, L. J., \& Barros, A. C. S. D. (2005). O impacto psicológico do câncer de mama. Revista Brasileira de Cancerologia, 51(2), 149-154.

Martin, L., Byrnes, M., McGarry, S., Rea, S., \& Wood, F. (2017). Posttraumatic growth after burn in adults: An integrative literature review. Burns, 43(3), 459470. doi: 10.1016/j.burns.2016.09.021

Nakayama, N., Mori, N., Ishimaru, S., Ohyama, W., Yuza, Y., Kaneko, T., ... Matsushima, E. (2016). Factors associated with posttraumatic growth among parents of children with cancer. PsychoOncology, 26(9), 1369-1375. doi: 10.1002/pon.4307

Nolen-Hoeksema, S., Wisco, B. E., \& Lyubomirsky, S. (2008). Rethinking rumination. Perspectives on Psychological Science, 3(5), 400-424. doi: 10.1111/j.1745-6924.2008.00088.x

Parikh, D., De Ieso, P., Garvey, G., Thachil, T., Ramamoorthi, R., Penniment, M., \& Jayaraj, R. (2015). Post-traumatic stress disorder and post-traumatic growth in breast cancer patients: a systematic review. Asian Pacific Journal of Cancer Prevention, 16(2), 641-646. doi: 10.1080/03630242.2012.679337

Park, C. L. (2005). Religion and meaning. Em F. Paloutzian \& C. L. Park (Eds.), Handbook of the psychology of religion and spirituality (pp. 295-314). New York: Guilford Press.

Pat-Horenczyk, R., Perry, S., Hamama-Raz, Y., Ziv, Y., Schramm-Yavin, S., \& Stemmer, S. M. (2015). Posttraumatic growth in breast cancer survivors: Constructive and illusory aspects. Journal of Traumatic Stress, (28), 214-222. doi: 10.1002/jts.22014

Pietrzak, J., \& Tsai, R. H. (2017). Trajectories of posttraumatic growth among US military veterans: A 4-year nationally representative, prospective cohort study. Acta Psychiatr Scand, 136(5), 483-492. doi: 10.1111 /acps.12800
Prati, G., \& Pietrantoni, L. (2009). Optimism, social support, and coping strategies as factors contributing to posttraumatic growth: A meta-analysis. Journal of Loss and Trauma, 14(5), 364-388. doi: 10.1080/15325020902724271

Ramos, C., Leal, I., Costa, P. A., Marôco, A. L., Guimarães, R., Fougo, J. L., \& Tedeschi, R. G. (2018). The effectiveness of a group intervention to facilitate posttraumatic growth among women with breast cancer. Psychooncology, 27(1), 258-264. doi: 10.1002/pon.4501

Rzeszutek, M., \& Gruszczyńska, E. (2018). Posttraumatic growth among people living with HIV: A systematic review. Journal of Psychosomatic Research, 114,81-91.doi:ht10.1016/j.jpsychores.2018.09.006

Schuettler, D., \& Boals, A. (2011). The path to posttraumatic growth versus posttraumatic stress disorder: Contributions of event centrality and coping. Journal of Loss and Trauma, 16(2), 180-194. doi: $10.1080 / 15325024.2010 .519273$

Seidl, E. M. . F., Tróccoli, B. T., \& Zannon, C. M. L. C. (2001). Análise fatorial de uma medida de estratégias de enfrentamento. Psicologia Teoria e Pesquisa, 17(3), 225-234. doi: 10.1590/S0102-37722001000300004

Seligman, M., \& Csikszentmihalyi, M. (2014). Positive psychology: An introduction. In Flow and the Foundations of Positive Psychology. Dordrecht: Springer.

Silva, T. L. G. da, Donat, J. C., Gauer, G., \& Kristensen, C. H. (2016). Posttraumatic growth measures: Translation and adaptation of three self-report instruments to Brazilian Portuguese. Archives of Clinical Psychiatry, 43(3), 47-50. doi: 10.1590/0101-60830000000083

Silva, T., Ramos, V., Donat, J., Oliveira, F., Gauer, G., \& Kristensen, C. (2018). Psychometric properties of the posttraumatic growth inventory in a sample of Brazilian university students. Trends in Psychiatric and Psychotherapy, 40(4), 292-299. doi: 10.1590/2237-6089-2017-0050

Stanton, A. L., Bower, J. E., \& Low, C. A. (2006). Posttraumatic growth after cancer. Em L. G. Calhoun \& R. G. Tedesch (Eds.), Handbook of posttraumatic growth: Research o practice (pp. 138-175). Mahwah, NJ, US: Lawrence Erlbaum Associates Publishers.

Taku, K., Cann, A., Tedeschi, R. G., Calhoun, L. G., Taku, K., Cann, A., ... Calhoun, L. G. (2015). Core Psico-USF, Bragança Paulista, v. 26, n. 3, p. 417-428, jul./set. 2021 
beliefs shaken by an earthquake correlate with posttraumatic growth. Psychological Tranma: Theory, Research, Practice and Policy, 7(6), 563-569. doi: $10.1037 / \operatorname{tra} 0000054$

Tedeschi, G. R., \& Calhoun, G. L. (1996). The posttraumatic growth inventory: Measuring the positive legacy of trauma. Journal of Traumatic Stress, 9(3), 455-471. doi: 0894-9867/96/0700-0455\$09.50/1

Tedeschi, R. G., \& Calhoun, L. G. (2004). Posttraumatic growth: Conceptual foundations and empirical evidence. Psychological Inquiry, 15(1), 1-18. doi:10.1207/ s15327965pli1501_01

Vieira, C. P., Lopes, M. H. B. de M., \& Shimo, A. K. K. (2007). Sentimentos e experiências na vida das mulheres com câncer de mama. Revista $D a$
Escola de Enfermagem Da USP, 311-6(2), 311-316. doi: 10.1590/S0080-62342007000200020

Vitalino, P. P., Russo, J., Carr, J. E., Maiuro, R. D., \& Becker, J. (1985). The ways of coping checklist: Revision and psychometric properties. Multivariate Behavioral Research, 20(1), 3-26. doi: 10.1207/ s15327906mbr2001_1

Zhou, N., Yu, W., Tang, S., Wang, J., \& Killikelly, C. (2018). Prolonged grief and post-traumatic growth after loss: Latent class analysis. Psychiatry Research, 267, 221-227. doi: 10.1016/j.psychres.2018.06.006

Recebido em: 09/04/2019

Reformulado em: 24/04/2020

Aprovado em: 07/09/2020 
Nota dos autores:

Os autores agradecem à Coordenação de Aperfeiçoamento de Pessoal de Nível Superior (CAPES) pela bolsa de Doutorado concedida aos dois primeiros autores.

Sobre os autores:

João Oliveira Cavalcante Campos é Psicólogo pela Universidade Federal do Ceará (UFC), mestre em psicologia pela Universidade Federal do Rio Grande do Sul (UFRGS) e especialista em terapias cognitivo-comportamentais (InTCC). Atualmente, é doutorando no Instituto de Psicologia da UFRGS.

ORCID 0000-0001-7471-2236

E-mail: joaoocc@hotmail.com / joaoocc18@gmail.com

Clara Cela de Arruda Coelho é Psicóloga pela Universidade Federal do Ceará (UFC), mestre e doutoranda em psicologia pela Universidade Federal do Rio Grande do Sul (UFRGS).

ORCID 0000-0002-8155-4712

E-mail: claracela@gmail.com

Clarissa Marceli Trentini é Psicóloga e mestre em psicologia pela Pontifícia Universidade Católica do Rio Grande do Sul (PUCRS), doutora em Ciências Médicas: Psiquiatria pela Universidade Federal do Rio Grande do Sul (UFRGS) e professora dos cursos de graduação e pós-graduação no Instituto de Psicologia da UFRGS.

ORCID 0000-0002-2607-7132

E-mail: clarissatrentini@terra.com.br

Contato com os autores:

João Oliveira Cavalcante Campos

Ramiro Barcelos, 2600, Sala 225

Porto Alegre-RS, Brasil

CEP: 90035-003

Telefones: 33085475 / (51) 982555198 